\title{
Surgical Technique in Pedicled Thoracodorsal Artery Perforator Flaps: A Clinical Experience with 99 Patients
}

\begin{abstract}
Moustapha Hamdi, M.D., Ph.D.

Koenraad Van Landuyt, M.D., Ph.D.

John B. Hijjawi, M.D.

Nathalie Roche, M.D. Phillip Blondeel, M.D., Ph.D. Stan Monstrey, M.D., Ph.D. Gent, Belgium

Background: The thoracodorsal artery perforator flap is considered a technically difficult flap because of significant anatomical variations in perforator location. The authors' strategy to facilitate the harvest of these flaps includes careful preoperative mapping of perforators and a standardized planning and harvesting technique. The authors evaluated 99 pedicled thoracodorsal artery perforator flaps, with an emphasis on preoperative planning, surgical technique, and analysis of complications.

Methods: Between May of 2000 and October of 2006, 99 patients underwent pedicled thoracodorsal artery perforator flaps in the authors' department. Their charts and postoperative results were reviewed retrospectively.

Results: A thoracodorsal artery perforator flap was harvested in 90 cases. The perforators were unsuitable in the other 10 flaps; therefore, a muscle-sparing technique was used (type I or type II). Unidirectional Doppler imaging was used exclusively in 92 percent of cases to map the perforator preoperatively. The average flap size was $20 \times 8 \mathrm{~cm}$. Average operative time for flap harvest was 80 minutes. Perforators were located at 8 to $13 \mathrm{~cm}$ from the axillary crease (average, $10.8 \mathrm{~cm}$ ). Major flap necrosis occurred in one case (1 percent). Limited partial or palpable fat necrosis occurred in three cases ( 3 percent). Seroma formation was not encountered in any of the thoracodorsal artery perforator flaps or muscle-sparing thoracodorsal artery perforator type I flaps.

Conclusion: Careful preoperative perforator mapping and a standardized approach to flap planning and harvest can significantly reduce the difficulty of executing pedicled thoracodorsal artery perforator flaps. (Plast. Reconstr. Surg. 121: 1632, 2008.)
\end{abstract}

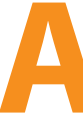

s the perforator flap concept has gained acceptance in the reconstructive ladder, many previously described musculocutaneous flaps have been harvested as perforator flaps. ${ }^{1-12}$ Although the thoracodorsal artery perforator flap has been reportedly used in multiple reconstructive situations, it has not gained the popularity of other perforator flaps such as the deep inferior epigastric artery perforator flap. Reasons for this include inconsistent perforator size, quality, quantity, and location, all of which contribute to tedious dissection. The lack of data

\footnotetext{
From the Plastic Surgery Department, Gent University Hospital.

Received for publication February 7, 2007; accepted March 27, 2007.

Portions of the video provided with this article were presented at the American Society of Reconstructive Microsurgery 2007 Meeting, in Rio Grande, Puerto Rico, January of 2007. Copyright $(02008$ by the American Society of Plastic Surgeons

DOI: $10.1097 /$ PRS.0b013e31816c3bfa
}

demonstrating a clear advantage of thoracodorsal artery perforator flaps over the more straightforward and time-tested latissimus dorsi flap or other local flaps such as scapular/parascapular flaps has

Disclosure: None of the authors has a financial interest in any of the products, devices, or drugs mentioned in this article.

Supplemental digital content is available for this article. Direct URL citations appear in the printed text; simply type the URL address into any web browser to access this content. Clickable links to the material are provided in the HTML text and PDF of this article on the Journal's Web site (www.PRSJournal.com). 
also contributed to criticism of the thoracodorsal artery perforator flap. ${ }^{4,11,12}$ Thus, only small clinical series of thoracodorsal artery perforator flaps have been published. ${ }^{1-12}$ The thoracodorsal artery perforator flap has been used widely in our department as either a free ${ }^{9}$ or a pedicled flap. ${ }^{6}$

The purpose of this article is to report our clinical experience with a large series of pedicled thoracodorsal artery perforator flaps used for a variety of indications, with an emphasis on preoperative planning, safe and standardized dissection technique, and an analysis of major and minor postoperative complications. In addition, a detailed video of the harvest technique is provided [see Video, Supplemental Digital Content 1, which demonstrates the harvest technique (described below), http://links. lww.com/A413].

\section{The Harvest Technique}

\section{Flap Design}

The anterior border of the latissimus dorsi muscle is marked with the patient in the standing position. Preoperative perforator mapping is performed with unidirectional Doppler imaging or by any of the other radiologic methods.

To anticipate possible septal cutaneous branches of the thoracodorsal artery, the anterior border of the flap should lie in front of the anterior border of the latissimus dorsi muscle. The vertically oriented flap can reach the distal third of the upper arm and elbow, and the neck, shoulder, and upper back area. When the thoracodorsal artery perforator flap is oriented horizontally, the incisions exploit the relaxed skin tension lines of the back. The flap is typically designed as in the classic latissimus dorsi musculocutaneous flap for breast reconstruction but is outlined more anteriorly to reach the lateral border of the inframammary fold.

\section{Patient Position}

The patient is in the lateral decubitus position with 90 degrees of shoulder abduction and 90 degrees of elbow flexion. This positioning places the skin on stretch, causing the perforators to enter the skin with a more perpendicular orientation, which makes their Doppler signal more discrete. Flap harvesting starts once the recipient site is ready. Dissection is performed under loupe magnification.

\section{Skin Incision}

Incision starts at the anteroinferior border of the flap, which allows identification of the anterior border of the latissimus dorsi muscle and the possibility of repositioning the anterior border of the flap accordingly. Then, the distal border of the flap is incised up to the area where the perforator signal has been detected.

\section{Suprafascial Dissection}

A caudal (posterior) approach is used in harvesting a pedicled thoracodorsal artery perforator flap, developing a plane above the deep fascia of the latissimus dorsi and serratus anterior muscles, which is thick medially and becomes thinner above the anterior part of the latissimus dorsi muscle. Only visibly pulsatile perforators with a diameter greater than $0.5 \mathrm{~mm}$ are considered suitable and preserved.

\section{Perforator Dissection}

Once a suitable perforator has been identified, the cleavage plane of the latissimus dorsi muscle in which that perforator resides is developed. Perforators originating from the descending branch of the thoracodorsal vessels are chosen preferentially over those originating from the transverse branch. Muscle fibers are spread, maintaining their longitudinal integrity. The perforator is dissected from the surrounding muscle fibers, where they tend to lie in a fibrofatty layer. Wide exposure is essential to perforator dissection within the muscle. The dissection should be performed close to the pedicle, ligating muscular branches with surgical clips or bipolar cautery. Nerve branches to the latissimus dorsi muscle should be freed from the pedicle with atraumatic dissection technique.

\section{Thoracodorsal Pedicle Dissection}

The main thoracodorsal pedicle is dissected free until the required pedicle length is obtained. Dissection of the main pedicle begins through the split muscle fibers. After the dissection of the main pedicle is performed, the segment of the perforator at the level where it enters the subcutaneous tissue is dissected from the latissimus dorsi muscle with the help of vessel loops.

\section{Completing the Flap Dissection}

The flap is then dissected from the serratus fascia and from the latissimus dorsi muscle anteriorly. Intercostal perforators, which may be encountered in front of the anterior border of the latissimus dorsi muscle, are clipped. The flap is then based totally on the thoracodorsal perforator and separated from the underlying muscles.

The flap is pulled through the muscle and transposed into the defect. As such, its anterior border becomes the medial or inferior part of the reconstruction. 


\section{PATIENTS AND METHODS}

Between May of 2000 and October of 2006, 99 patients underwent pedicled flaps based on the thoracodorsal vessels in our department. One patient had bilateral flaps; thus, 100 flaps are included in this study. The medical charts were reviewed retrospectively for information regarding patient characteristics, flap dimensions, the number and location of perforators, operative time, and complications. The average patient age was 44 years (range, 17 to 69 years) and 89 percent were female patients. Active smoking history was found in 11 patients (all presenting for immediate breast reconstruction for breast cancer). Surgical indications are summarized in Table 1 . The results show data regarding flap type, number and locations of perforators, and operative time. Postoperative complications are presented with a comparison among the different flap types.

\section{Flap Design}

The anterior border of the latissimus dorsi muscle is palpated through the skin and marked with the patient in the standing position and both hands grasping the waist. Preoperative perforator mapping is performed with a unidirectional Doppler probe $(8 \mathrm{~Hz})$ with the patient in the lateral decubitus position to simulate operative positioning. Based on previous anatomical studies, the perforators are sought out in a region $8 \mathrm{~cm}$ below the axillary crease (i.e., an axillary wrinkle found at the junction of the upper arm with the shoulder/back region) and within $5 \mathrm{~cm}$ of the anterior border of the latissimus dorsi muscle. ${ }^{1} \mathrm{~A}$ possible pitfall of using unidirectional Doppler imaging to identify the thoracodorsal perforators is background signal from the main thoracodorsal

\section{Table 1. Indications for a Total of 100 Pedicled Thoracodorsal Artery Perforator Flaps}

\begin{tabular}{lc}
\hline Recipient Site/Indication & $\begin{array}{c}\text { No. of } \\
\text { Pedicled Flaps }\end{array}$ \\
\hline Chest wall & 73 \\
Immediate partial breast reconstruction & 5 \\
Secondary partial breast reconstruction & 7 \\
Mastectomy & 2 \\
Salvage free flap breast reconstruction & 3 \\
Aesthetic breast augmentation & 2 \\
Sarcoma resection & 4 \\
Axilla & 2 \\
Contracture following burn & \\
Hidradenitis & 1 \\
Shoulder & 1 \\
Sarcoma resection & \\
Acute burn & \\
\hline
\end{tabular}

pedicle. To avoid this, the patient is positioned for perforator marking in the same position in which they will be placed during surgery: the lateral decubitus position with 90 degrees of shoulder abduction and 90 degrees of elbow flexion. This positioning places the skin on stretch, causing the perforators to enter the skin with a more perpendicular orientation, which makes their Doppler signal more discrete. In this orientation, the Doppler device detects the signal from the discrete end of the perforator rather than along the length of the perforator. This allows one to distinguish the perforator signal from the longitudinally oriented thoracodorsal pedicle. True perforator tones can be distinguished from the thoracodorsal artery by moving the Doppler device proximally and distally. If the signal disappears, the signal belongs to a perforator, although signals from the thoracodorsal vessels will be present continuously along the length of the latissimus dorsi muscle. In difficult cases, a duplex examination is performed. More recently, multidetector row computed tomography has been introduced for the preoperative localization of various perforators. ${ }^{13,14}$

To anticipate possible septal cutaneous branches of the thoracodorsal artery, the anterior border of the flap should lie in front of the anterior border of the latissimus dorsi muscle. Depending on the indication, the flap is outlined as a vertical ellipse or horizontally over the latissimus dorsi muscle. The vertically oriented flap can reach the distal third of the upper arm and elbow, and the neck, shoulder, and upper back area. The thoracodorsal artery perforator flap can also be harvested as a bilobed flap with a large longitudinal and a smaller transversely oriented skin island which, after a 90-degree rotation, allows for easier primary closure. When oriented horizontally, the incisions exploit the relaxed skin tension lines of the back. The flap is typically designed as in the classic latissimus dorsi musculocutaneous flap for breast reconstruction but it is outlined more anteriorly to reach the lateral border of the inframammary fold. The main indications are in breast surgery, either in partial breast reconstruction after ablative surgery ${ }^{6}$ or in autologous augmentation. ${ }^{7}$ The flap reaches the superolateral, inferolateral, and inferomedial quadrants of the breast. Flaps $15 \mathrm{~cm}$ wide can be raised safely on a single perforator, depending on the recipient-site requirements and the ability to achieve primary closure of the donor site. 


\section{Surgical Technique}

When a pedicled thoracodorsal artery perforator flap is harvested, the patient is positioned as described above (see Video, Supplemental Digital Content 1, which demonstrates the harvest technique, http://links.lww.com/A413). Flap harvesting starts once the recipient site is ready. Dissection is performed under loupe magnification. Incision starts at the anterointerior border of the flap, which allows identification of the anterior border of the latissimus dorsi muscle and the possibility of repositioning the anterior border of the flap accordingly. Then, the distal border of the flap is incised up to the area where the perforator signal has been detected. A caudal (posterior) approach is used in harvesting a pedicled thoracodorsal artery perforator flap, developing a plane above the deep fascia of the latissimus dorsi and serratus anterior muscles, which is thick medially and becomes thinner above the anterior part of the latissimus dorsi muscle. Only perforators that are visibly pulsatile with a diameter greater than $0.5 \mathrm{~mm}$ are considered suitable and preserved. The main perforators tend to lie in a row overlying the course of the descending branch of the thoracodorsal artery.

Once a suitable perforator has been identified, the cleavage plane of the latissimus dorsi muscle in which that perforator resides is developed. Perforators originating from the descending branch of the thoracodorsal vessels are chosen preferentially over those originating from the transverse branch because they typically have a shorter intramuscular course with less involvement of nerve branches. More distal perforators result in a longer pedicle than perforators entering the flap more proximally or cranially. The disadvantage of more distally located perforators is that dissection requires more time because of their longer intramuscular course. Muscle fibers are spread, maintaining their longitudinal integrity. The perforator is dissected from the surrounding muscle fibers, where they tend to lie in a fibrofatty layer. Wide exposure is essential to perforator dissection within the muscle. The dissection should be performed close to the pedicle, ligating muscular branches with surgical clips or bipolar cautery. Nerve branches to the latissimus dorsi muscle should be freed from the pedicle with atraumatic dissection technique.

The main thoracodorsal pedicle is dissected free until the required pedicle length is obtained. Dissection of the main pedicle begins through the split muscle fibers and is then completed under the latissimus dorsi muscle by lifting its anterior border. The flap is pulled through the muscle and transposed into the defect. As such, its anterior border becomes the medial or inferior part of the reconstruction.

\section{Conversion to Muscle-Sparing Designs}

When the perforator is less than $0.5 \mathrm{~mm}$ in diameter but pulsatile, there is a high risk for perforator rupture or avulsion. In this situation, a muscle-sparing technique (muscle-sparing thoracodorsal artery perforator type I) increases safety by preserving a 2-cm cuff of latissimus dorsi muscle around the perforators. The perforators are still dissected free of the latissimus dorsi muscle anteriorly, but their posterior aspect remains attached to a cuff of muscle, which is included in the flap. Including this small segment of latissimus dorsi muscle while maintaining direct visualization of the perforators reduces the potential for damaging them during repositioning of a pedicled thoracodorsal artery perforator flap. Only nerve branches to this muscle segment need to be sacrificed.

When the perforators are smaller than $0.5 \mathrm{~mm}$ and also nonpulsatile, the flap should be converted to a muscle-sparing thoracodorsal artery perforator type II flap incorporating a maximum number of perforators. An approximately 5-cm segment of latissimus dorsi muscle is included under the skin paddle between the cleavage plane where the perforators are identified and the anterior border of the latissimus dorsi muscle, with a length extending to the level of the main pedicle. The nerve supplying the remaining latissimus dorsi muscle is preserved.

Depending on the nature of the defect, the flap is partially or totally deepithelialized and can also be folded to increase projection. However, tension-free flap inset is critical to avoid rupture of the perforator. All patients receive piracetam (Nootropil; UCB, Brussels, Belgium) $12 \mathrm{~g} / 24$ hours intravenously for 5 days and as a $20 \%$ solution orally, $25 \mathrm{cc}$ four times per day for another 5 days, to improve the viability of the distal flap through an increase of capillary blood flow.

\section{RESULTS}

The majority of patients underwent preoperative perforator mapping with unidirectional Doppler examination (92 percent). Both color duplex and multidetector row computed tomographic images were used in 4 percent of patients for preoperative perforator identification. The average flap size was $20 \times 8 \mathrm{~cm}$ (range of length, 16 

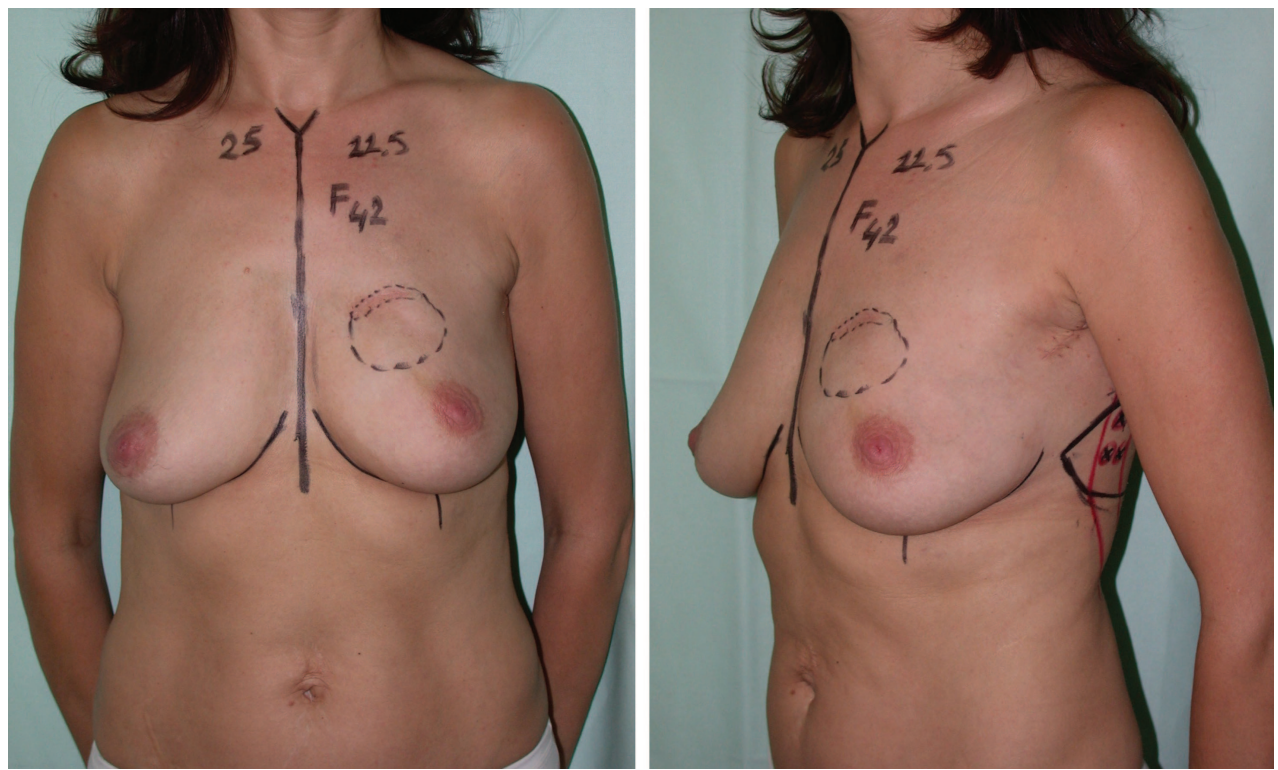

Fig. 1. Preoperative views of a 42-year-old woman with failure of breast-conserving therapy for left breast cancer in the superomedial quadrant.

to $25 \mathrm{~cm}$; range of width, 6 to $10 \mathrm{~cm}$ ). Ninety thoracodorsal artery perforator flaps were harvested. Muscle-sparing thoracodorsal artery perforator type I and type II flaps were dissected in six and four cases, respectively. Thoracodorsal artery perforator and muscle-sparing thoracodorsal artery perforator type I flaps (96 flaps) were based on a single perforator in 89 cases (92.7 percent) and on two perforators in seven cases $(7.3$ percent). In these flaps, most of the perforators originated from the descending branch of the thoracodorsal vessels (93.7 percent), and only six flaps were harvested on a perforator from the transverse branch (6.3 percent). At least two perforators were included in the flap (four flaps) when a musclesparing thoracodorsal artery perforator type II flap was harvested. The thoracodorsal artery perforator flap and its muscle-sparing variants are demonstrated with clinical examples in Figures 1 through 8 .

Perforators were located between 8 and $13 \mathrm{~cm}$ (average, $10.8 \mathrm{~cm}$ ) distal to the axillary crease and within 0 to $5 \mathrm{~cm}$ (average, $2.8 \mathrm{~cm}$ ) of the anterior border of the latissimus dorsi muscle. In two cases, the thoracodorsal artery perforator flap was based on a direct septal perforator that went around the anterior border of the latissimus dorsi muscle rather than through the muscle. The average harvesting time was 80 minutes (range, 25 to 120 minutes).

Postoperative complications are summarized in Table 2. Total flap survival was achieved in 96 cases (96.7 percent). Major flap necrosis occurred in one case (1 percent), which was an immediate partial breast reconstruction. This required debridement and secondary flap surgery. Limited partial or palpable fat necrosis occurred in three cases (3 percent), requiring partial excision in one case. None of these complications was related to

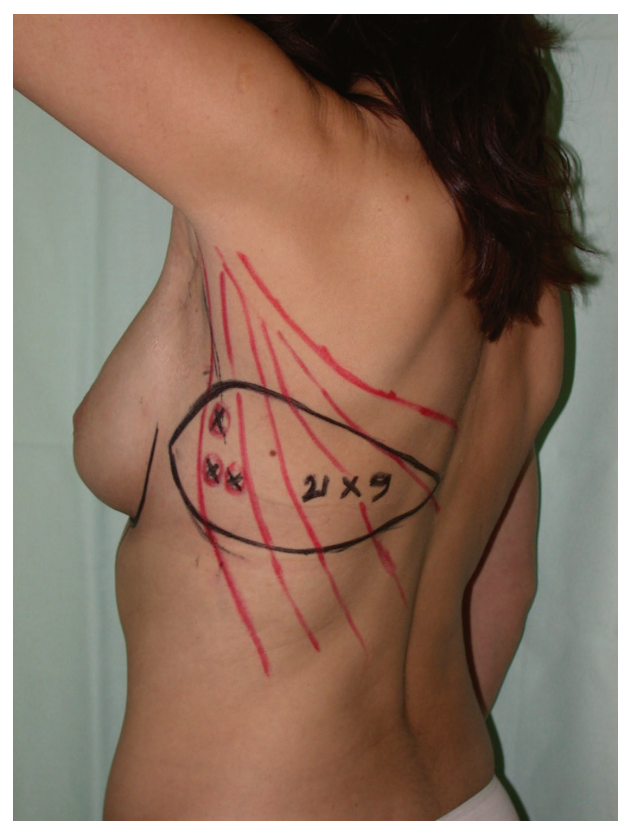

Fig. 2. A pedicled $(21 \times 9-\mathrm{cm})$ thoracodorsal artery perforator flap was designed with a horizontal orientation with three perforators detected by means of a unidirectional Doppler probe. 


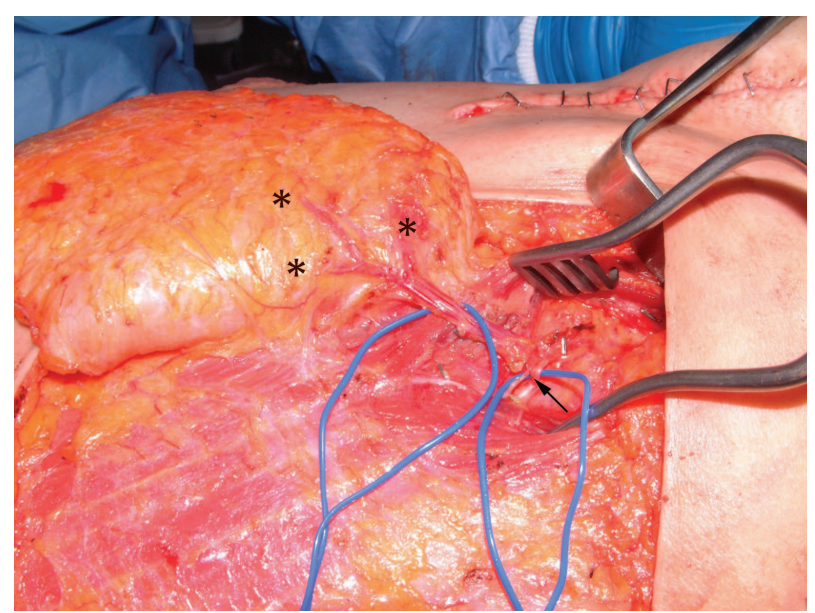

Fig. 3. Intraoperative view shows the location of the perforators (asterisks), which correspond to the Doppler findings. Two perforators (one with a bifurcation) were dissected. The thoracodorsal nerve branches (arrow) to the latissimus dorsi muscle are spared.

smoking history. However, all three flaps that suffered partial necrosis were irradiated postoperatively. Seroma formation at the donor site was encountered in all cases of muscle-sparing thoracodorsal artery perforator type II flaps but in none of the thoracodorsal artery perforator or musclesparing thoracodorsal artery perforator type I flaps.

\section{DISCUSSION}

The thoracodorsal artery perforator flap is a versatile tool in reconstructive surgery, and flaps as large as $25 \times 15 \mathrm{~cm}$ can be safely harvested. .,2,8 $^{2}$ Several anatomical variations can make the harvest of thoracodorsal artery perforator flaps tech-

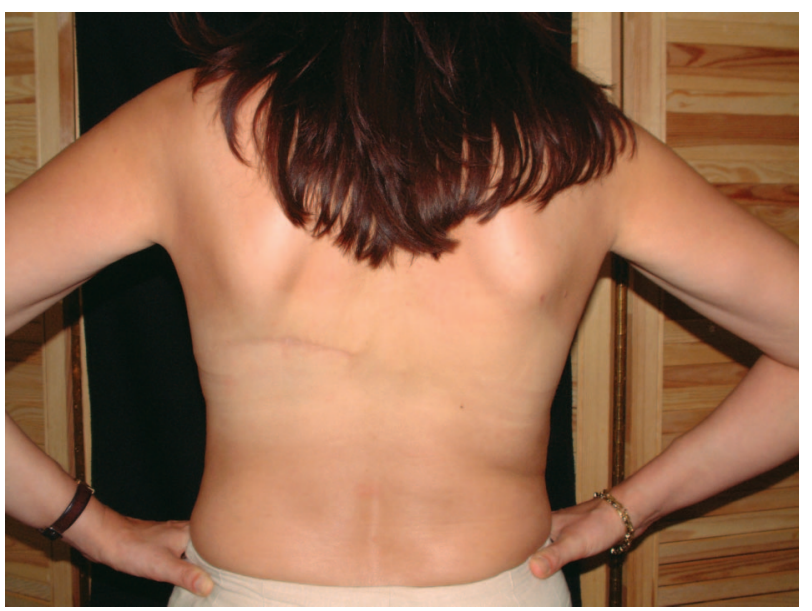

Fig. 5. The donor site.

nically challenging. These include a variable number of large $(>0.5 \mathrm{~mm})$ perforators; difficulty mapping these perforators preoperatively because of the proximity of the main thoracodorsal pedicle; and poorly described, sometimes tedious dissection techniques. Lastly, there is a lack of convincing data demonstrating a clear advantage of the thoracodorsal artery perforator flap over other pedicled flaps such as latissimus dorsi muscle or parascapular flaps.

The thoracodorsal vessels provide limited large perforators to the overlying skin. ${ }^{3,7,15,16}$ Recent studies demonstrate only $5.5 \pm 1.8$ perforators with a diameter greater than $0.5 \mathrm{~mm}$ supplying this flap. ${ }^{15}$ However, only one or two perforators can be clinically used if a useful arc of rotation is to be maintained. Multiple cadaveric dissection studies have focused on determining the relationship between topographic landmarks and the thoracodorsal perforators. ${ }^{2,8,15}$ Since the original description of
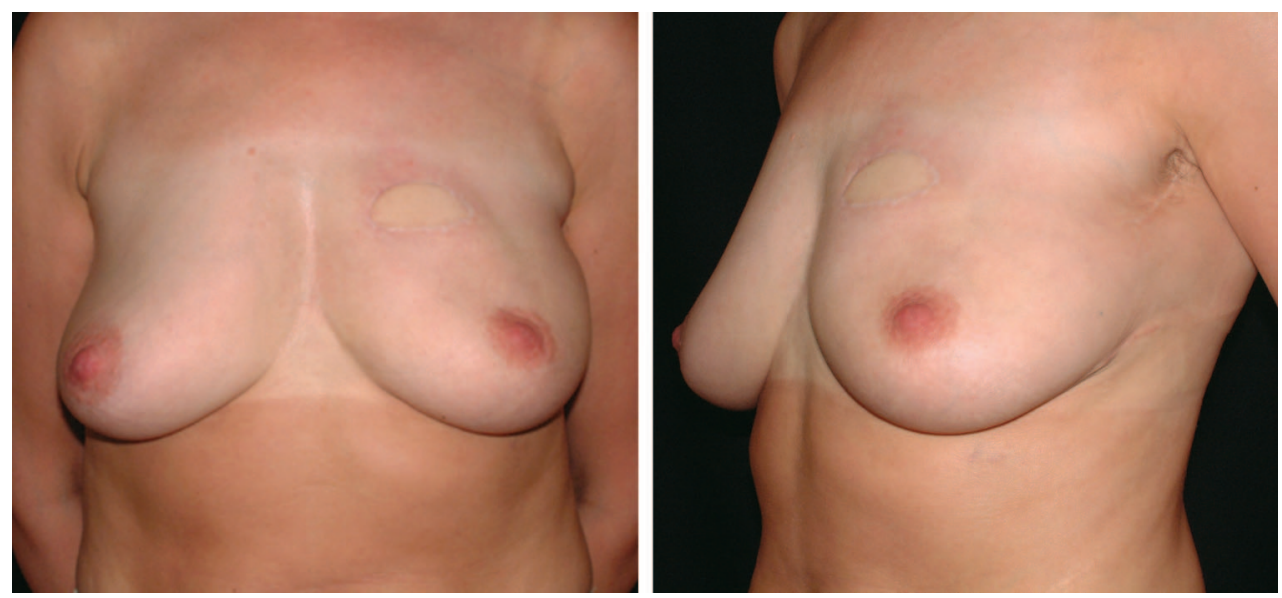

Fig. 4. Postoperative views. 

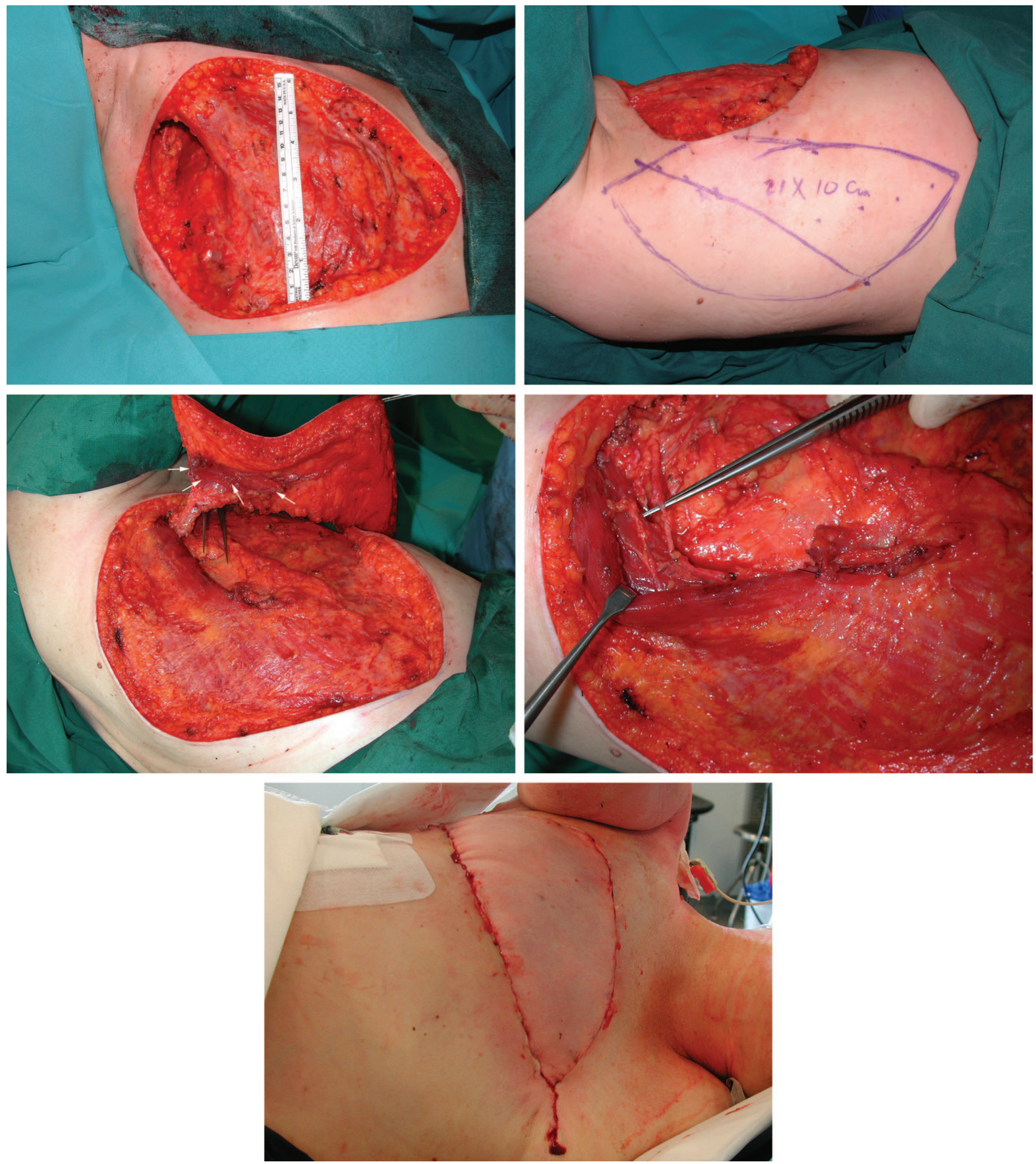

Fig. 6. A case of mastectomy for carcinomatous mastitis. (Above, left) The $24 \times 15-\mathrm{cm}$ defect. (Above, right) A pedicled thoracodorsal artery perforator flap $(21 \times 10 \mathrm{~cm})$ was planned with a vertical orientation. (Center, left) A pedicled musclesparing thoracodorsal artery perforator type I flap was harvested with a latissimus dorsi muscle segment measuring $4 \times 2$ $\mathrm{cm}$ (arrows). (Center, right) The thoracodorsal nerve branches to the remaining muscle were spared. (Below) Closure of the donor site.

the thoracodorsal artery perforator flap by Angrigiani et al., ${ }^{1}$ the location of the majority of perforators $8 \mathrm{~cm}$ distal to the posterior axillary fold remains a valid and important anatomical landmark. At this point, the first perforator emerges from the descend- ing branch of the thoracodorsal pedicle and usually travels above the deep fascia caudally and parallel to the latissimus dorsi muscle fibers. The scapular tip was used by Heitmann et al. ${ }^{5}$ to locate the bifurcation of the thoracodorsal vessel into its respective hori- 

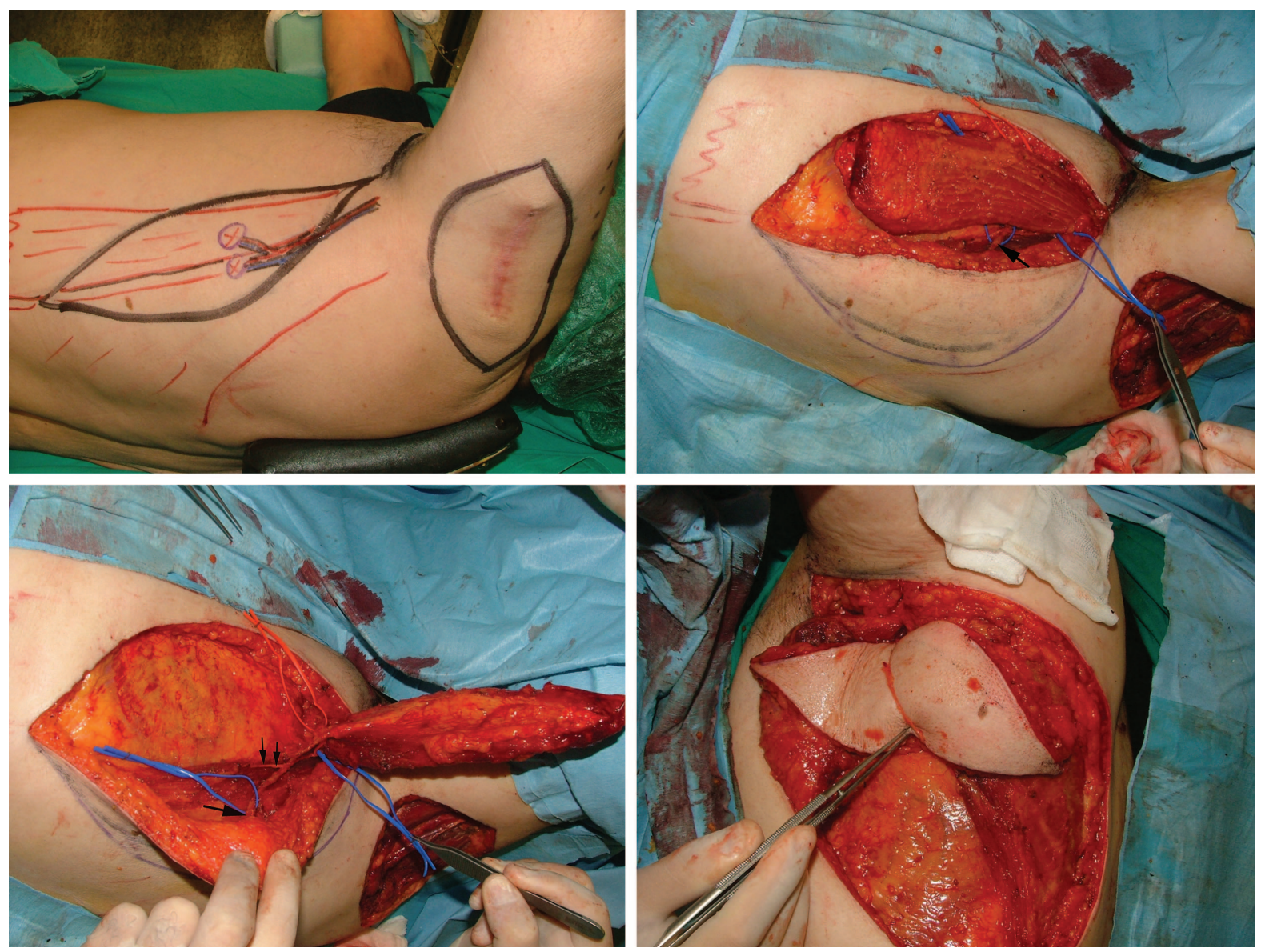

Fig. 7. A case of sarcoma on the left shoulder of a 56-year-old man. (Above, left) A compound (chimera) flap is designed. The flap consists of a thoracodorsal artery perforator flap with a segment of latissimus dorsi muscle to reconstruct the deltoid muscle because of tumor resection. (Above, right) The latissimus dorsi segment $(10 \times 5 \mathrm{~cm})$ was dissected with a vascular pedicle independent of the pedicle of the thoracodorsal artery perforator flap (arrow). (Below, left) The muscle segment is elevated. The perforator arises from the horizontal branch and courses under the thoracodorsal nerve branch (two arrows) to enter the skin paddle (vessel loop and arrow). (Below, right) The thoracodorsal artery perforator flap is passed under the thoracodorsal nerve that is left intact to the rest of the latissimus dorsi muscle.

zontal and lateral branches. The neurovascular hilus was found 3 to $6 \mathrm{~cm}$ inferior to the scapular tip and 1 to $4 \mathrm{~cm}$ posterior to the anterior border of the latissimus dorsi muscle. These landmarks help to determine an area in which large perforators can be found consistently. However, this area depends on the position of the patient and laxity of the skin and may not correspond to intraoperative findings. ${ }^{16}$ Consequently, significant time can be spent searching for skin perforators and modifying the flap design. ${ }^{16}$

The ability to safely and expeditiously harvest a thoracodorsal artery perforator flap is facilitated by precise preoperative localization of its perforators. Using one of the several available radiologic assessment methods mentioned provides information specific to an individual pa- tient, making flap harvest safer and faster. Unidirectional Doppler examination is a simple and inexpensive method for mapping perforators but is somewhat less accurate than color Doppler flowmetry. ${ }^{17}$ Placing the patient in an operative position is recommended, to increase the accuracy of all perforator mapping techniques. We have used the Doppler probe in all of our cases, with reliability as high as 95 percent. However, color duplex flowmetry and multidetector row computed tomography have been shown to facilitate dissection, particularly in inexperienced hands. ${ }^{13,14}$ These recent imaging developments allow us to localize the "dominant perforator" and obtain complete information about the arterial tree of the thoracodorsal vessels and intercostal perforators, thus decreasing opera- 


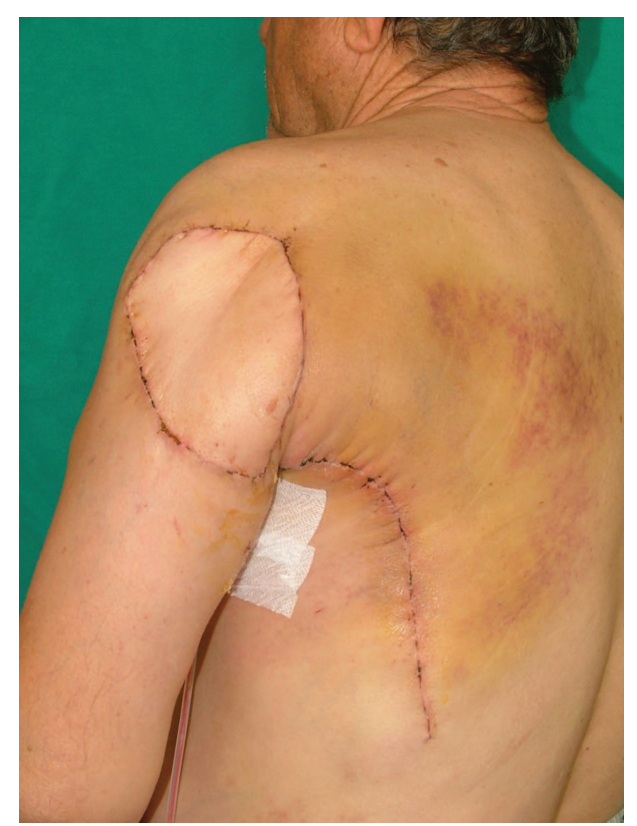

Fig. 8. Early postoperative result, with uneventful wound healing.

Table 2. Complications after Harvesting Pedicled Thoracodorsal Artery Perforator and Muscle-Sparing Type I and II Flaps

\begin{tabular}{lccc}
\hline Complications & $\begin{array}{c}\text { TDAP } \\
(\boldsymbol{n}=\mathbf{9 0})\end{array}$ & $\begin{array}{c}\text { MS-TDAP } \\
\text { Type I } \\
(\boldsymbol{n}=\mathbf{6})\end{array}$ & $\begin{array}{c}\text { MS-TDAP } \\
\text { Type II } \\
(\boldsymbol{n}=\mathbf{4})\end{array}$ \\
\hline $\begin{array}{l}\text { Major partial loss } \\
\text { Partial flap loss/fat }\end{array}$ & 1 & 0 & 0 \\
$\quad$ necrosis & 2 & 0 & 1 \\
Wound dehiscence & 1 & 1 & 1 \\
Seroma & 0 & 0 & 4 \\
\hline
\end{tabular}

TDAP, thoracodorsal artery perforator; MS, muscle-sparing.

tive time and complications related to poor perforator selection.

Another major advantage of the thoracodorsal artery perforator is pedicle length. With an average length of $14 \pm 1.4 \mathrm{~cm}$, the flap can reach more medial defects on the chest wall than the scapular/ parascapular flap. ${ }^{8,15}$ When the thoracodorsal artery perforator flap is based on a distal perforator, pedicle length can reach $25 \mathrm{~cm},{ }^{8}$ providing a broad arc of rotation extending over the anterior chest wall, clavicle, axilla, posterior arm, and lateral border of the sternum. Tension on the pedicle must be avoided, as this may lead to rupture of smaller perforators. Alternative flaps such as those based on the intercostal or superior epigastric vessels are more suitable for defects of the medial chest wall. ${ }^{18}$

Initial dissection begins with an anterior, caudal incision and identification of the anterior bor- der of the latissimus dorsi muscle. Making the anterior, caudal incision first allows the remaining skin paddle to be adjusted to guarantee inclusion of perforators that course anterior to the latissimus dorsi edge. The caudal approach allows safe visualization of the perforator because the elevation of the skin is performed parallel to the latissimus dorsi fibers and the perforator can be seen through transparent fascia. The perforator lies in loose areolar tissue within a cleavage plane in the latissimus dorsi muscle, facilitating dissection. Regular irrigation of the perforator with saline and delicate manipulation is recommended to avoid spasm. Spasm usually resolves spontaneously once blood in the main pedicle is redirected entirely into the perforator after ligation of side branches. If spasm persists, topical papaverine irrigation can be used. Failure of spasm to resolve typically indicates intraluminal damage of the perforator intima because of excessive traction during dissection and usually results in flap failure. We recommend keeping the posterior aspect of the perforator attached to the latissimus dorsi muscle where the perforator enters the skin until just before flap transfer.

Flap-related complications in this study were limited. Partial flap necrosis occurs if the flap is extended beyond it vascular territory, estimated at $255 \mathrm{~cm}$ by Thomas et al. ${ }^{15}$ In comparing our experience with a vertical flap design (used mainly in microvascular transfer) and a more horizontal flap orientation (used mainly in pedicled flaps), we have observed a slightly higher rate of partial necrosis, typically in the distal tip, in the vertical design. This is likely related to the orientation of the angiosome territory of the thoracodorsal perforators. According to Taylor's findings, ${ }^{19}$ flaps would most reliably be designed parallel to the course of the ribs in this region. This favors a horizontal, slightly oblique orientation of the flap, with its tip directed toward the scapular angle. This design was used in the majority of our pedicled thoracodorsal artery perforator flaps for breast reconstruction, giving the additional advantages of donor-site closure within the relaxed skin tension lines and scar placement within the bra area.

Although latissimus dorsi muscle preservation is an intuitive benefit that results in less contour deformity of the donor site than latissimus dorsi harvest, prospective studies are still necessary to confirm the objective functional benefits of latissimus dorsi muscle sparing. Nevertheless, this study does confirm that harvesting thoracodorsal artery perforator or muscle-sparing thoracodorsal 
artery perforator type I flaps dramatically reduces the incidence of seroma formation in the donor site, which has been reported to be as high as 60 percent after harvesting a latissimus dorsi muscle flap. ${ }^{20}$ In addition, shorter postoperative recovery and less pain at the donor site have been reported after harvesting other perforator flaps. ${ }^{21}$

\section{CONCLUSIONS}

This study demonstrates that pedicled thoracodorsal artery perforator flaps can be harvested safely to cover a wide range of defects. Careful surgical planning by preoperative perforator mapping is essential for successful harvesting of the thoracodorsal artery perforator flap. Using the surgical technique described here provides a safe approach to avoid complications related to flap design or dissection. We feel that perforator flaps can safely replace the classic latissimus dorsi muscle/musculocutaneous flap in many clinical situations, with an attendant reduction in morbidity to the patient. Muscle harvest can frequently be reserved for procedures requiring functional or dynamic reconstruction. Reducing complications at both the donor and recipient sites should remain a constant goal in reconstructive surgery.

Moustapha Hamdi, M.D.

Plastic Surgery Department

Gent University Hospital

De Pintelaan 185

B-9000 Gent, Belgium

moustapha.hamdi@rug.ac.be

\section{REFERENCES}

1. Angrigiani, C., Grilli, D., and Siebert, J. Latissimus dorsi musculocutaneous flap without muscle. Plast. Reconstr. Surg. 96: 1608, 1995.

2. Spinelli, H. M., Fink, J. A., and Muzaffar, A. R. The latissimus dorsi perforator-based fasciocutaneous flap. Ann. Plast. Surg. 37: 500, 1996.

3. Koshima, I., Saisho, H., Kawada, S., Hamanaka, T., Umeda, N., and Moriguchi, T. Flow-through thin latissimus dorsi perforator flap for repair of soft-tissue defects in the legs. Plast. Reconstr. Surg. 103: 1483, 1999.

4. Schwabegger, A. H., Bodner, G., Ninkovic, M., and PizaKatzer, H. Thoracodorsal artery perforator (TAP) flap: Report of our experience and review of the literature. Br. J. Plast. Surg. 55: 390, 2002.

5. Heitmann, C., Guerra, A., Metzinger, S. W., Levin, L. S., and Allen, R. J. The thoracodorsal artery perforator flap: Anatomic basis and clinical application. Ann. Plast. Surg. 51: 23, 2003.
6. Hamdi, M., Van Landuyt, K., Monstrey, S., and Blondeel, P. Pedicled perforator flaps in breast reconstruction: A new concept. Br. J. Plast. Surg. 57: 531, 2004.

7. Van Landuyt, K., Hamdi, M., Blondeel, P., and Monstrey, S. Autologous breast augmentation by pedicled perforator flaps. Ann. Plast. Surg. 53: 322, 2004.

8. Guerra, A. B., Metzinger, S. E., Lund, K. M., Cooper, M. M., Allen, R. J., and Dupin, C. L. The thoracodorsal artery perforator flap: Clinical experience and anatomic study with emphasis on harvest techniques. Plast. Reconstr. Surg. 114: 32, 2004.

9. Van Landuyt, K., Hamdi, M., Blondeel, P., and Monstrey, S. The compound thoracodorsal perforator flap in the treatment of combined soft-tissue defects of sole and dorsum of the foot. Br. J. Plast. Surg. 58: 371, 2005.

10. Laredo-Ortiz, C., Salvador-Sanz, J., Marquez Mendoza, M., et al. Pedicled thoracodorsal arterial perforator flap for reconstruction of a large defect post-burn in posterior side of the arm. Burns 31: 108, 2005.

11. Er, E., and Ucar, C. Reconstruction of axillary contractures with thoracodorsal perforator island flap. Burns 31: 726, 2005.

12. Rehman, N., Kannan, R. Y., Hassan, S., and Hart, N. B. Thoracodorsal artery perforator (TAP) type I V-Y advancement flap in axillary hidradenitis suppurativa. Br. J. Plast. Surg. 58: 441, 2005.

13. Masia, J., Clavero, J. A., Larranaga, J. R., Alomar, X., Pons, G., and Serret, P. Multidetector-row computed tomography in the planning of abdominal perforator flaps. J. Plast. Reconstr. Aesthet. Surg. 59: 594, 2006.

14. Hamdi, M., Van Landuyt, K., Van Hedent, E., and Duyck, P. Advances in autogenous breast reconstruction: The role of preoperative perforator mapping. Ann. Plast. Surg. 58: 18, 2007.

15. Thomas, B. P., Geddes, C. R., Tang, M., Williams, J., and Morris, S. F. The vascular basis of the thoracodorsal artery perforator flap. Plast. Reconstr. Surg. 116: 818, 2005.

16. Lin, C. T., Huang, J. S., Yang, K. C., Hsu, K. C., Chen, J. S., and Chen, L. W. Reliability of anatomical landmarks for skin perforators of the thoracodorsal artery perforator flap. Plast. Reconstr. Surg. 118: 1376, 2006.

17. Tsukino, A., Kurachi, K., Inamiya, T., and Tanigaki, T. Preoperative color Doppler assessment in planning of anterolateral thigh flaps. Plast. Reconstr. Surg. 113: 241, 2004.

18. Hamdi, M., Van Landuyt, K., de Frene, B., Roche, N., Blondeel, P., and Monstrey, S. The versatility of the intercostal artery perforator (ICAP) flaps. J. Plast. Reconstr. Aesthet. Surg. 59: 644, 2006.

19. Taylor, G. I. The angiosomes of the body and their supply to perforator flaps. Clin. Plast. Surg. 30: 331, 2003.

20. Randolph, L. C., Barone, J., Angelats, J., Dado, D. V., Vandevender, D. K., and Shoup, M. Prediction of postoperative seroma after latissimus dorsi breast reconstruction. Plast. Reconstr. Surg. 116: 1287, 2005.

21. Kroll, S. S., Sharma, S., Koutz, C., et al. Postoperative morphine requirements of free TRAM and DIEP flaps. Plast. Reconstr. Surg. 107: 338, 2001. 